\section{Strength of Metal Single Crystals}

IN the course of some experimental work on single crystals of cadmium, which I have been carrying out under Prof. E. N. da C. Andrade, I have found that the condition of the surface exercises a surprisingly large effect on the initial strength of the crystal. It is well known that glide commences in a single crystal when the shear stress on the glide plane, and in the glide direction, reaches a certain value, known as the critical shear stress. Thus in a series of crystals grown from cadmium (glide plane, hexagonal base; glide direction, axis of digonal symmetry), which proved to contain $0 \cdot 15$ per cent of lead and 0.15 per cent of zinc, this critical shear stress lay within the comparatively narrow range of $50-59 \mathrm{gm}$. wt. per sq. mm., while the angles which the hexagonal planes made with the axis of the wire varied from $8^{\circ}$ to $72^{\circ}$.

When the crystals are grown in the presence of oxygen, there is a marked increase in the resistance to shear, values as high as $120 \mathrm{gm}$. wt. per sq. $\mathrm{mm}$. being obtained. As neither nitrogen, carbon dioxide nor water vapour produces a similar effect, the increased resistance to shear is attributed to a film of oxide formed on the surface of the metal. Oxidation of the wires subsequent to their conversion into single crystals has the same effect, and even keeping the wires in a damp atmosphere at room temperature for several weeks produces a measurable effect. Removal of the oxide film by brushing the surface with dilute sulphuric acid reduces the critical shear stress to the normal value of about $60 \mathrm{gm}$. wt. per sq. $\mathrm{mm}$.

The effect cannot be attributed to any strength of the oxide film, since, first, there are considerations to show that it can only be a few atoms thick, and, secondly, there is a growth of the resistance to shear as deformation proceeds, and not the weakening which must ensue on rupture of the oxide film if its tensile strength were in question.

To eliminate the effect of impurities, and of surface film, cadmium was carefully purified by sublimation in vacuo, which left no lead that could be detected, and less than $0 \cdot 1$ per cent of zinc, and oxide-free crystals were grown from it. A single crystal prepared in this way showed a critical shear stress of only $13.7 \mathrm{gm}$. wt. per sq. $\mathrm{mm}$. This seems to confirm the hypothesis of Haase and Schmidt ${ }^{1}$ that crystals of perfectly pure metal would be unable to withstand even the smallest shear stress. It may be noted that A. W. Hanson ${ }^{2}$ has just published some results for very pure zinc, which bring the critical shear stress down to $9 \cdot 1 \mathrm{gm}$. wt. per sq. mm., but he does not refer to any influence of surface factors.

It seems clear, then, that the ideal metal lattice has very little or no strength, slip starting at the surface and proceeding inwards, and that surface films of a certain character can prevent initiation of slip, and so greatly strengthen the crystal. The analogy offered by the behaviour of rock salt, where the surface is of such significance, as evidenced by the behaviour under water, will readily occur.

Physics Laboratory,

R. Roscoe.

\section{University College, \\ London, W.C.1. \\ May 11.}

\footnotetext{
1 Z. Phys., 33, 413; 1925. See also H. J.Gough, D. Hanson, and J. J. Wright, Phil. Trans., A, 226, 1; 1924

Phys. Rev., 45, 324; 1934 .
}

Zostera Disease on the Coast of County Cork, I.F.S.

IN view of the widespread effects of Zostera (seagrass and eel-grass) disease and the interest which it has aroused, the following brief note appears to be worthy of publication. Zostera was first noticed to be affected by some sort of trouble in Castle Haven during the summer of 1932, when the extensive meadows near and north of Castletownshend were much less luxuriant than usual. (Just off Castletownshend the plants are usually extremely robust and of great length.) By the summer of 1933 the meadows had vanished. At Lough Ine the various beds were unaffected until late in 1933. By December those in fairly deep water between the Coosh and the mainland, in the southern region of Barloge Creek and the two large meadows in Southern's Bay just below the Rapids, which are exposed at very low water, were very worn in appearance. By the middle of February this year they had disappeared, as had those which formerly all but covered the floor of the inner part of the Goleen, except for their dead remains. At the end of March it was found that a further bed had completely disappeared from the Ballyally side of the Coosh. By this time new growth had started in the Goleen and very soon became vigorous, whilst by the end of April there was strong growth over large areas in Barloge Creek, but no sign of any in Southern's Bay.

Three chief points of interest emerge from these observations: the disease seems to be spreading very slowly along the south coast of Ireland, having taken two years to travel the six miles which separate Castle Haven from Barloge Creek: recovery has been extremely rapid : $Z$. marina, $Z$. nana and the hybrid were equally affected and have made equally rapid recoveries. All three occur in the Goleen, marina alone in the other localities. I am told that at Castle Haven there has been no recovery but a simultaneous decrease in flatfish.

University College,

Louis P. W. Renouf.

Cork.

May 22.

\section{Control of Chromatophores in Leander serratus}

THE phenomena of colour-change in prawns are well-known ${ }^{1,2}$, and the mechanisms controlling these phenomena understood in the main ${ }^{2,3}$. Certain details, however, require further explanation, and among them are the following :-

It is stated ${ }^{2}$ that for Palcemonetes varians, a form very similar to Leander, the effect of $(a)$ darkness and $(b)$ light on a white background, is the same; red and yellow chromatophores contract and reflect. ing yellow chromatophores expand. The reverse condition is produced by light on a dark background.

I find that in Leander, conditions $(a)$ and $(b)$ produce similar but not identical effects. The reflecting yellow chromatophores, fairly numerous in Leander, do not expand and contract automatically in a reverse direction to red and yellow types (which are under hormone control) but behave independently and according to light intensity. Thus, irrespective of the background, they expand in good light and contract in dim light or in darkness. They also continue to do this in eyeless animals where the red and yellow chromatophores are no longer under control. It is already known ${ }^{1,4}$ that in certain 